(Aus der Universitäts - Frauenklinik zu Leipzig. Director:

Geheimrath Zweifel.)

\title{
Ueber das Vordringen des Chorion laeve in die Tubenschleimhaut nebst Bemerkungen uiber die tubare Eieinbettung.
}

\author{
Von \\ Privatdocent Dr. H. Füth, \\ Assistenzarzt der Klinik. \\ (Hierzu Tafel $\mathrm{V}$ und 3 Abbildungen im Text.)
}

Meine bisherigen Arbeiten auf dem Gebiete der tubaren Eieinbettung haben sich vor Allem mit den Befunden am Eiboden beschäftigt und zu der Anschauung geführt, dass das Ei bei seiner Einbettung in der Tube activ vorgeht. Zwar ist auch das Schicksal der Eikapsel zur Sprache gekommen, aber doch nicht in der Weise für die Richtigkeit der obigen Anschauung verwerthet worden, wic es der Bedeutung der Sachlage entspricht und wie ich es in den nachfolgenden Zeilen zu thun beabsichtige. Thatsächlich besteht ein grosser Unterschied zwischen der Eikapsel im Uterus und in der Tube, und um den Gegensatz scharf hervorzuheben, schicke ich das über beide Feststehende kurz voraus.

Wir wissen, dass die junge Decidua capsularis alle Bestandtheile der Vera enthält: Drüsen, Capillaren, Deciduazellen ${ }^{1}$ ); aussen und innen fehlt ihr eine Epithelauskleidung. So findet man sie am Ende der ersten Schwangerschaftswoche. Nach weiteren 14 Tagen nimmt die Kapsel an Dicke zu: Drüsen, Capillaren und Deciduazellen treten klar zu Tage und der um das $\mathrm{Ei}$ geschlossene Ring besteht aus einem und demselben Gefüge. Mit dem Wachsthum des Eies atrophirt die Capsularis, doch finden sich in einer Text. S. 98. 
dicken Capsularis bis zum Ende des 4. Monats nach den Untersuchungen von Hofmeier auch bei normalen Verhältnissen noch deutlich Drüsen vor. Im 4. Monat legt sich die Capsularis an die epithellos gewordene Vera an, mit der sie verschmilzt; ist aber bis zum Ende der Schwangerschaft als schmaler Zellstreifen, in welchem sich Zottenreste eingestreut finden, fast in allen Präparaten noch erkennbar.

Ganz anders liegen die Verhältnisse in der schwangeren Tube. Zwar kommt es auch hier za einem gleieh vollkommenen Absehluss des Eies, wie neuerdings besonders $\mathrm{Krömer}{ }^{1}$ ) betont; allein eine Deridua capsularis wie im Uterus giebt es hier nicht. Die das Ei rom Tubenlumen absehliessende Membran bestcht aus den obersten abgespalteten Lagen der Schleimhaut, an die sich noch Sehleimhautfalten zur Verstärkung anlegen können. Sie ist unnachgiebig, starr, unelastisch, wic die Ausdrücke in den Beschreibungen lauten; sie wird rein passiv gedehnt und wic Werth) sagt, durch die Abspaltung von der gefässführenden Unterlage und den Contaet mit den trophoblastischen Zellmassen des darunterliegenden lies nekrotisch. Sehr bald wird sie dann von den Zellen des T'rophoblastes durchbrochen; dessen Zellen legen sich der gegenüberliegenden Schleimhaut der Tube direct an und dringen vor Allem in die der likuppe gegenüberliegenden Faltenkämme ein. Die erste genauere histologische Schilderung dieser Befunde ist, wie Werth hervorhebt, ron mir ausgegangen, während der Vorgang selber schon früher von Orthmann und \%edel, ja makroskopisch schon von Hennig beobachtet war, was ich damals übersehen hatte und nachträglich gerne anerkenne. Ebenso sind andere auf Grund eigener Untersuchungen za wesentlich denselben Ergebnissen gekommen, so vor Allem Aschoff3), Voigts), auch Petersen ${ }^{5}$, Erfolgt das Reissen der Capsularis, wie Veit in

1) Untersuchungen iaber die tubare Lieinbettung. Dieses Acchir. 68. Bd. S. 504 .

2) In seinem Referat über kixtrauteringraviditä zum Congress in Würzburg, Pfingston 1903. Verhandl. des 10. Congresses. S. 160.

3) In seinem ausgezeichneten Referat: Neue Arbeiten über die Anatomie und Aetiologie der 'l'ubenschwangerschaften. Centralbl. f, allgem. Pathol. u. pathol. Anat. 1901. Bd. XII. No. 11/12.

4) Zur Bildung der Capsularis in der 'lubenwand. Dieses Arehir. 68. Bd. 1903. H. 3.

5) Beiträge zur pathologischen Anatomie der 'l'ube. Berlin 1902. S. Karger, S. 52 . 
seinem Referat zum Würzbúrger Congress ausführt, bei sehr jugendlichen Thieren, so wird dabei der intervillöse Raum eröffnet und Blut strömt aus ihm in den Kanal der Tube, meist mit dem Ergebniss, dass das Ei verloren ist. Findet die Usur später statt, so fehlt die Blutung, die Frucht wird in ihrer Entwicklung nicht gestört, das Ei gewinnt Raum, sich in das Lumen der Tube hinein zu entwickeln, und das Chorion laeve liegt der gegenüberliegenden Schleimhaut der Tube unmittelbar an. Gerade hierauf möclite ich an der Hand eines mir geeignet erscheinenden Präparates etwas ausführlicher eingehen, welches ich makroskopisch bereits vor 2 Jahren in der Leipziger geburtshilfichen Gesellschaft demonstrirte ${ }^{1}$ ).

Es war damals einige Tage zuvor durch Laparotomie (Operateur Geheimrath $Z$ weifel) von einer 34 3. Pat, die glatt genas, gewonnen worden. Dieselbe war nach ihren Angaben bereits vier Jahre zuvor in Basel von Herrn Professor. Bumm wegen linksseitiger Eileitersehwangerschaft operirt worden. Obwohl diese Mittheilung in . vorstehender Form ganz bestimmt gemacht wurde, glaubte ich doch nachfragen zu sollen und bin Herrn Professor von Herff sehr zu Dank verpflichtet für seine liebenswürdige Auskunft, dass bei derselben Patientin am 16. November 1897 ein linksseitiger faustgrosser Fruchtsack, der theilweise geplatzt war, entfernt wurde. Sie hatte dann nachher ihre Periode regelmässig weiter gehabt bis zum 17. November 1901. Mitte Januar 1902 fiel sie von einem Wagen. Es trat darauf eine 4 Tage anhaltende starke Blutung ein, worauf wieder alles in Ordnung zu sein schien. Ende desselben Monats überhob sie sieh, es wurde ihr sehwarz vor den Augen und es trat ein heftiger Schmerz in der rechten Seite, sowie Drängen nach dem Mastdarme auf und sie wurde ohnmächtig. Seitdem bestehen starke Sehmerzen in der rechten Seite. Die Diagnose war leicht zu stellen und es fand sich nach Eröffnung der Bauchhöhle in dieser nur wenig dunkles flüssiges Blut. An der Stelle, die vom Tumor rechts zuerst sichtbar wurde, lagen diesem Blutcoagula auf.

Makroskopische Beschreibung. Nach Exstirpation der der rechten Tube angehörenden Geschwulst - das rechte Ovarium blieb zurück - ergab die Besichtigung des Präparates, welches etwa die Grösse einer mittleren Faust hatte, dass bei der Aus-

1) Centralbl. f. Gyn. 1902. S. 532. 
schälung die erwähnten Blutcoagula sich abgelöst hatten und Placentargewebe in einem etwa Zehnpfennigstück grossen Defecte auf der vorderen Wand frei zu Tage $\operatorname{lag}{ }^{1}$ ). Ein ähnlicher, kleinerer Defect in der Fruchtsackwand, aus dem ebenfalls Placentargewebe frei heraus ragte, befand sich correspondirend auf der hinteren Fläche. Fig. 1, Taf. V lässt dieses Hervortreten des Placentargewebes deutlich erkennen. (Es liegt also einer jener Ausnahme-. fälle vor, von denen Werth in seinem Referate spricht, dass sich nämlich hier und da auch an offene Rupturen nur ein in Gestalt einer Hämatocele sich begrenzender Bluterguss anschliesst.) Ferner sieht man das nach dem Uterus zu sich zuspitzende Ende des Sackes und das weit klaffende Fimbrienende, das zum Theil durch eine streifige, wohl aus geronnenem Blute hervorgegangene Masse verschlossen wird, während zum übrig bleibenden Theile des Ostiums wiederum Placentargewebe in ziemlich grosser Ausdehnung hervorquillt. Dicht neben der zuerst erwähnten Durchbruchsstelle der Placenta auf der vorderen Wand liegt abdominalwärts eine etwa 5 pfenniggrosse Stelle, die dunkel durehschimmert. Das ganze Präparat hatte zweierlei Consistenz. Bei Betastung der nahe der Abtrennungsstelle vom Lig. lat. zu gelegenen Parthie hatte man das Gefühl einer weichen Masse, während nach der entgegengesetzten Richtung, nach der convexen Fläche hin die dünne Wand deutlich schwappte. Man konnte etwas wie von einem Fötus durchfühlen und in der That zeigte das Röntgenbild das wohl ausgebildete Skelett eines Fötus. Nach sorgfältiger Härtung wurde das Präparat über die Mitte der oberen Fläche hinweg der Länge nach aufgeschnitten und man kam auf diese Weise in einen Sack hinein (Fig. 1), auf dessen Boden ein etwa 3 monatlicher Fötus lag, der nicht die geringsten Zeichen dafür aufwies, dass er bereits längere Zeit abgestorben gewesen wäre. Der Sack war von einer feinen Membran ausgekleidet, die nirgendwo eine lücke aufwies (Amnion). In demselben befand sich flockig geronnener Inhalt.

1) Das Hervorquellen von Placentargewebe durch einen Defect in der Wand eines extrauterinen Fruchtsackes findet man in sehr schöner Weise wiedergegeben bereits in Fig. 9 (bei b), Tafel XXI des geburtshilflichen Atlas ron H. F. Kilian. Es handelt sich hier allerdings um eine Graviditas interstitialis und, wie es in der Beschreibung heisst; ,man erbliclst auf diesem Tumor einen kleinen Riss, durch welchen hindurch sich einige Gefässbüschel des Chorion gedrängt haben i. Herr Geheimrath $Z$ weifel machte mich auf diese interessante Abbildnng aufmerksam. 
Ein grösseres Gerinnsel lag dicht dem uterinen Ende der Tube an und hier inserirte ganz marginal auch die Nabelschnur. Die Placenta, auf deren stark concar ausgebogene Fläche man von oben herabsieht, war deutlich als solche zu erkennen. Sie nimmt den Boden des Sackes ein und erstreckt sich aufwärts in unregelmässiger Begrenzung bis $c$ und $c^{\prime}$, stellenweise noch etwas höher. Ich zerlegte dann das Präparat in zahlreiche Querscheiben und Fig. 2 stellt eine solche von der abdominalen Seite aus gesehen dar.

Figur 1.

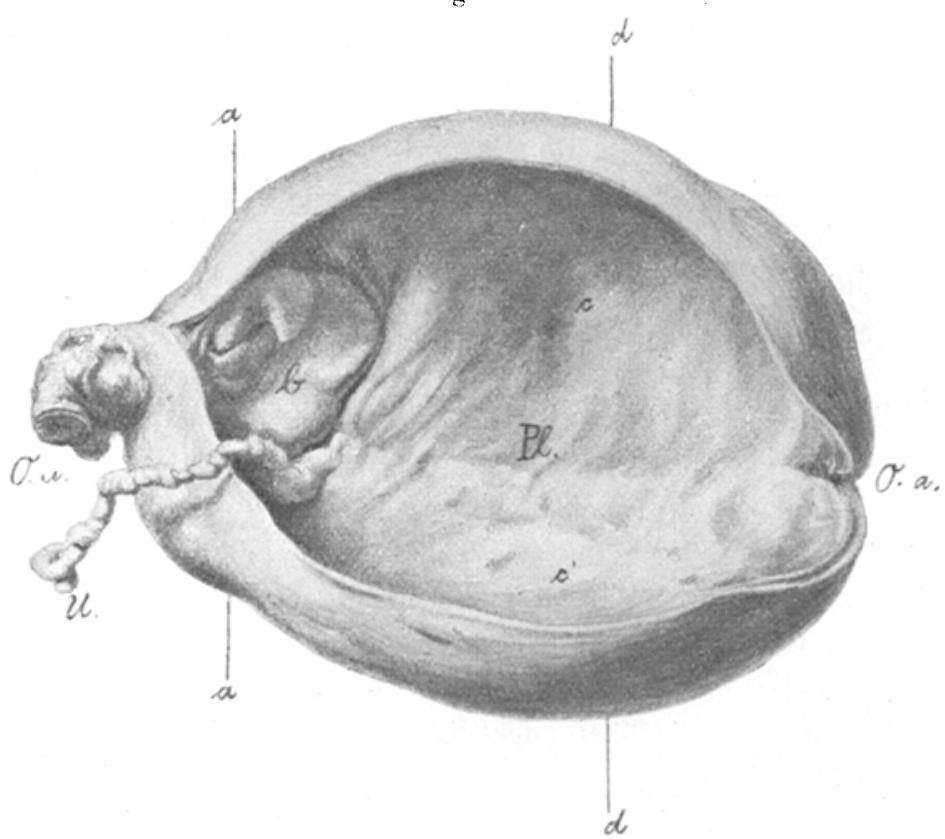

Die Eihöhle mit Placenta und Nabelschnur. 0. u., 0. a. Ostium uterinum, abdominale, U Nabelschnur, Pl Placenta, am Boden des Sackes liegend, seitlich bis $c$ und $\mathrm{c}^{\prime}$ aufwärts reichend, a grenzt die Querscheibe ab, welche, uterinwärts beginnend, in Serienschnitten untersucht wurde, b ein grosser geronnener Klumpen (Niederschlag aus dem Fruchtwasser), d bezeichnet die Stelle, welcher die in Fig. 2 wiedergegebene Querseheibe entspricht.

Man sieht vor Allem die Durchbruchsstelle der Wand und das Hervortreten der Placenta, deren Ausbreitung, sowie die Amnioshöhle.

Mikroskopische Beschreibung. Die Querseheiben dicht hinter dem Ostium uterinum beginnend, bis etwa an a in Fig. 1 habe ich nach Celloidineinbettung in Serienschnitte zerlegt und zur Färbung zum Theil die van Gieson'sche, zum Theil Haematoxylin- 
Figur 2.

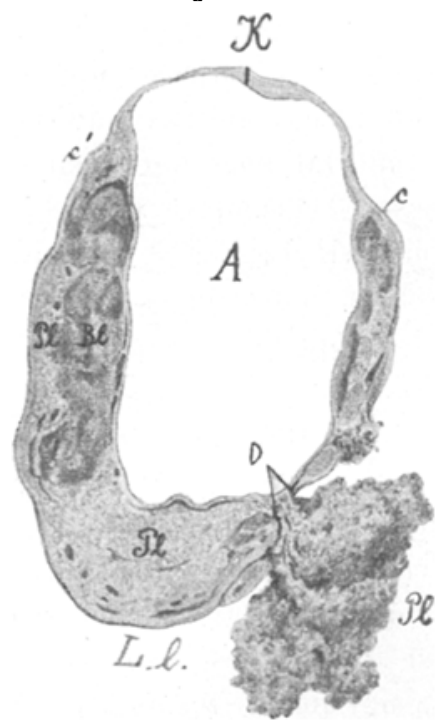

Querseheibe, ron der Fläche aus gesehen, entsprechend d in Fig. 1. D Durchbruchsstelle des Fruchthalters, Pl Placentargewebe, A Amnioshöhle, Bl Blutcoagula, K Kıppe des Präparates, reconstruirt (vergl. Fig. 1), L I Ansatzstell des ligamentum latum, e $e^{i}$ wic in Tig. 1.

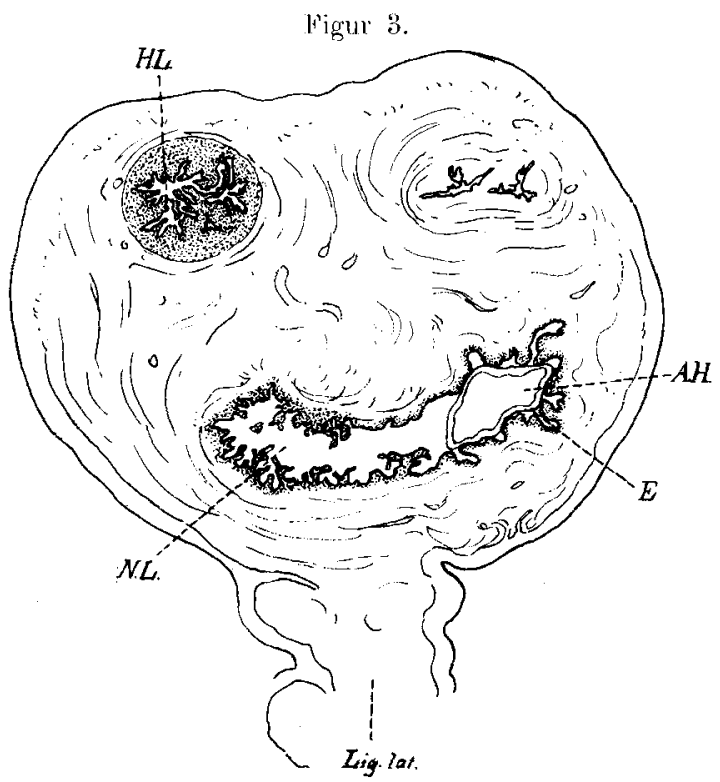

Querschaitt durch die schwangerc Tube, da, wo Eihäute und Amnionhöhle in dem Nebentubenlumen N I zu erscheinen beginnen. H I Haupttubenlumen, F Chorionepithel, A H Amnioshöhle und Amnionepithel, Iig. lat. Ansatzstelle des Jigamentum latum. 
Eosin benutzt, habe dann aber noch eine Reihe Schnitte von andern Querscheiber angelegt und diese in gleicher Weise gefärbt.

1. Aus dem Studium der Serienschnitte ergab sich Folgendes: In den ersten, vom uterinen Ende an numerirten Schnitten zeigte sich ein Tubenlumen mit zahlreich verzweigten Falten, das Epithel cubisch, niedrig. Dieses Tubenlumen lässt sich weiterhin als geschlossenes Hauptlumen (H L, Fig. 3) verfolgen. Vielfach zeigen sich die Falten desselben unter einander verschmolzen und das umgebende Gewebe aufgequollen und gelockert. Sehr oft sieht man die Mucosazellen zu typischen Deciduazellen umgewandelt. In der Umgebung dieses Hauptlumens fanden sich eine Reihe kleiner Lumina, die sich auf Serienschnitten als Abzweigungen vom Hauptlumen darstellten und kamen und wieder verschwanden ${ }^{1}$ ). Pon Schnitt 69 an erscheinen dann die Anfänge eines zu einem grossen Nebentubenlumen sich entwickelnden Ganges, der von dem Hauptlumen zunächst streng geschieden ist (Fig. 3, N L). Dagegen sind die kleinen Lumina rechts oben in der Fig. 3 Ausläufer dieses Nebentubenganges, wie sich aus den Serienschnitten unzweifelhaft ergiebt. Von Schnitt 120 an beginnt der Fruchtsack sich zu zeigen (vergl. Fig. 3), man bemerkt deutlich Chorion, Amnion und Amnioshöhle. Die vorher schon vorhanden gewesene typische deciduale Umwandlung der Mucosazellen beschränkt sich weiterhin auf die Abschnitte des Nebentubenganges, welche in der von Eihäuten freien Ecke liegen. Die Gefässe in der Umgebung dieses Nebentubenganges, namentlich in der Richtung nach dem Lig. lat. zu sind mächtig entwickelt, in ihrem Innern enthalten sie oft Zellen und geben ein Bild, wie ich es Taf. V, Fig. 11 im

1) Eine eingehende Würdigung haben diese Gebilde kürzlich in einer Arbeit von Robert Meyor gefunden: "Ueber adenomatöse Schleimhautwucherungen in der Uterus- und Tubenwand und ihre pathologisch-anatomische Bedeutung." Virchow's Archiv, 1898, Bd. 172, S. 404, heisst es: „In manchen Fällen ist dieses Hervorgehen aus der Schleimhaut kaum oder gar nicht mehr kenntlich, weil der Zusammenhang unterbrochen wird; trifft man nun alle Schichten der Tubenwand mit Drüsen durchsetzt, ohne ihren Zusammenhang mit der Schleimhaut nachweisen zu können, so liegt die Versuchung nahe, an eine anderweitige Herkunft der Drüsen zu denken. Jedoch habe ich mich überzengt, dass ein einziger enger Schleimhautausläufer zahllose Verzweigungen in alle Wandschichton senden kann und zwar auf grosse Strecken. Es macht dieser Befund Angesichts des wenig veränderten Tubenlumens einen ausserordentlich merkwürdigen Eindruck; er erinnert an eine Wasserleitung, in deren Röhren das Wasser unter hohem Druck steht; durch die kleinste Oeffnung spritzt das Wasser aus und bildet eine Fontäne." 
63. Bande dieses Archivs wiedergegeben habe. Der Peritonealüberzug zeigt die bekannten, von Werth zuerst beschriebenen Wucherungen und darunter deciduaähnliche Umwandlung der subperitoncalen Bindegewebszellen. Im weiteren Verlaufe der Serie ergiebt sich dann, dass das Nebentubenlumen sich immer mehr ausdehnt, der Umfang der Eihäute wird grösser, die Amnioshöhle weiter, die Entfernung bis zum Hauptlumen immer kürzer, bis schliesslich ein Uebergang in den Hauptgang statthat und dessen Lumen seitlich dem Eiraum ansitzt, wie in Fig. 3 der mit N 1. bezeichnete Abschnitt des Nebentubenganges. Allmälig wird dann das ganze Tubenlumen in den Bereich der immer grösser werdenden Eihöhle hineinbezogen und entsprechend den Abschnitten, denen in Fig. 2 die Placenta anliegt, beginnen sich Chorionzotten zu zeigen, die vereinzelt dem peritonealen Ueberzuge sebr nahe rücken. Auf der Eikuppe ( $K \mathrm{coc}^{\prime}$ in Fig. 2) breitet sich das Chorion laeve aus. Hier findet sich Tubenschleimbaut stellenweise noch wohl erhalten vor. Die Wand selbst ist auf eine ganz dümne lage faserigen Gewebes reducirt. Reste einer Capsularis habe ich weder auf dem von Chorionzotten freien Abschnitte der Eikuppe, noch in dem Nebentubengange feststellen können.

Von ganz besonderem Interesse ist meines Erachtons die Art und Weise, wie das Chorion laeve in dem Nebentubengang sowic weiter nach Verschmelzung desselben mit dem Hauptlumen bis zum Auftreten von Zotten sich gegenüber dem Tubenlumen und besonders dem Tubenepithel verhält. Da ist einmal auffallend die ausserordentlich starke Faltenbildung der Eihäute, offenbar als Ausdruck dafür, dass mit der Vergrösserung ihrer Oberfläche die Ausdehnung der Fruchtsackwand nicht hat Schritt halten können. Eine Andeutung dieser Fältelung findet sich in Fig. 3, Taf. V, bei c. Es zeigt hier das Chorion eigenthümliche Einbuchtungen; je weiter aber man sich der Mitte des Fruehtsackes näbert, um so grösser werder die Faltenbildungen, an denen sich dann Amnion und Chorion in gleicher Weise betheiligen. Weiter gestaltet sich das Verhalten des Chorionepithels gegenüber dem Tubenepitbel ganz auffallend. Fig. 2-4, Taf. V, erklären dies besser als jede Beschreibung. Ich füge deshalb nur ganz kurz hinzu: Es giebt sehr wenig Strecken, auf denen das T'ubenepithel fehlt und die Eihänte sich der nackten Tubenschleimhaut anlegen. An vielen Stellen laufen Tuben- und Chorionepithel neben einander her, wie in Fig. 2 zu sehen ist. Beide Epithelarten unterscheiden sich sehr deutlich 
von einander; die Kerne der Chorionepithelien sind mehr rundlich und färben sich blass; die Kerne der Tubenepithelien sind mehr länglich und meist tiefdunkel gefärbt. An anderen Stellen legen sich die Chorionepithelien auf die Kuppen der zahlreich vorspringenden Tubenfalten auf und die Grenzen zwischen beiden verwischen sich (vergl. Fig. 3 bei a und b). Betrachtet man dann eine Stelle wie $b$ bei starker Vergrösserung, so erhält man ein Bild, wie es in Fig. 4 wiedergegeben ist. Man sieht, dass die blasser gefärbten Chorionepithelien sich keilförmig zwischen die auseinander gedrängten Tubenepithelien einschieben und so in das subepitheliale Bindegewebe eindringen, dessen Kerne stellenweise eine deutliche deciduale Umwandlung aufweisen. An Schnitten, die weiter nach der Mitte des Eies zu liegen, zeigt sich ein ganz eigenthümliches Bild. Hier hat sich das Chorion ganz um eine vorspringende Tubenfalte herumgelegt, während das Tubenepithel zu Grunde gegangen ist. Man bekommt dadurch ein Bild, das an die Haut mit ihren Papillen erinnert, insofern ein epithelialer Zellmantel eine bindegewebige Papille (das bindegewebige Stroma der Tubenschleimhauterhebung) umgiebt. Da. wo das Chorionepithel sich in das subepitheliale Bindegewebe einsenkt, finden sich mächtig entwickelte Gefässe, sowie zahlreiche Hämorrhagien, an welch letztere die Chorionepithelien hier und da heranreichen. Etwas anders liegen die Verhältnisse auf der $\mathrm{Ei}$ kuppe, insofern ( $\mathrm{K}$ in Fig. 2) in Folge der Spannung die Epithelien sehr flach, fast endothelartig geworden sind und die gegenseitige Beziehung zu einander nicht so klar zu Tage tritt wie in dem Nebentubengang. Die Schleimhaut weist hier kaum noch Vorsprünge auf, die Eihäute liegen meist unmittelbar dem subepithelialen Bindegewebe auf und das Tubenepithel findet sich nur auf ganz kurze Strecken erhalten. Dieser Scilderung möchte ich noch hinzufügen, dass ich sämmtliche Befunde, ausgenommen die an der Eikuppe, an Serienschnitten studirt habe, insbesondere aber das Eindringen der Chorionepithelien in die Schleimhaut, so dass Trugbilder durch Schrägschnitte u. dergl. ausgeschlossen sind.

Einer kritischen Besprechung schicke ich voraus, dass mit dem activen Vordringen des Eies unter die tabare Schleimhaut sich auf Grund der Graf Spee'schen Untersuchungen am Meerschweinchen, sowie der Peters'schen Befunde an einem jungen Ei neuerdings viele von denen befreundet haben, welche dieser Auffassung Anfangs ablehnend gegenüberstanden. Anders ist es da- 
gegen mit der Ansicht, dass das Ei auch weiterhin in der Tube bei seiner Einbettung astiv thätig sei. Denn das activ unter die Sehleimhaut gelangte Meerschweinchenei verhält sich weiterhin durchaus passiv. Wie die schönen Untersuchungen von Hermann und Stolper ${ }^{1}$ ) dargethan haben, ist die nach erfolgter Einnistung secundär vor sich gehende Tiefenlagerung des Meerschweincheneies ein für das $\mathrm{Ei}$ passiver Vorgang, und diese Beobachtung analog für die Einbettungsvorgänge des menschlichen Eies nicht zu verwerthen, liegt kein Grund vor. Denn der Uterus wächst mit dem sich vergrössernden $\mathrm{Ei}$ bis zum 4 . Monate in ausgedehntem Maasse mit; er lässt das $\mathrm{Ei}$ in eine weiche, nachgiebige, mächtig entwickelte Decidua sich einbetten; er macht es durch die ausnehmend grosse Erweiterung seiner Capillaren, die $\mathrm{His}^{2}$ ) eingehend geschildert hat, dem Eie leicht, sieh in die für sein Fortkommen genügende Berührung mit den mütterlichen Blutbahnen zu setzen. Das alles fällt in der Tube für gewöhnlich fort, wie ich schon früher genau auseinandergesetzt habe ${ }^{3}$, und deshalb muss auch die weitere Einbettung des Eies, wenn es in seiner Entwicklung vorwärts kommen will, nothwendigerweise eine andere sein, als im Uterus. Daran ändern auch nichts die neueren Beobachtungen von Micholitsch, der etwa in 30 Fällen nahezu regelmässig Nebentubengänge fand und angiebt, dass or fast jedesmal die Einbettung des Eies in einem Nebentubengang habe nachweisen können. Doch sagt $\mathrm{Werth}^{3}$ ) in der kritischen Besprechung dieser Arbeit, dieselbe lïsst die Möglichkeit einer anderen Deutung der Befunde, namentlich einer secundären Hineinbeziehung benachbarter Gänge in das ursprünglich vom Hauptlumen aus angelegte Eibett, nicht ganz ansgeschlossen erscheinen. Zu Befunden, die sich mit denen von Micholitsch zum Theil decken, ist dann neuerdings 0 . Fellner ${ }^{4}$ ) gekommen. Er untersuchte in dem laboratorium von Wertheim drei 2-3 Wochen alte Tubeneier in Serienschnitten und kommt zu dem Ergebniss, dass die Divertikeleinbettung nichts etwas Zufälliges, sondern der T'ubargravidität eigenthümlich sei. 0 . Fellner trug hierüber auf der Naturforscherversammlung in Cassel vor und in der Discussion wiesen Veit und Heinsius seine Auf-

1) Münch. med. Wochenschr. 1903. S. 1230.

2) Dieses Archiv. 63. Bd. 1901. S. $141 \mathrm{ff}$.

3) 1. e. S. 168 .

4) Zur Frage der Divertikeleinbettung in der Tube. Minch. med. Wochenschr. 1903. S. 1893. 
fassung zurück, worauf Fellner im Schlusswort betonte, er wolle nicht behaupten, dass die Divertikelbildung die einzige Ursache für die Entstehung der Tubargravidität sei. Auf die Bedeutung dieser Divertikel für die Eieinbettung an sich habe ich schon in meiner ersten ${ }^{1}$ ) Arbeit hingewiesen. Ich sagte damals: „Göbel streitet beiderlei Arten von Gängen sozusagen die Möglichkeit ab, ätiologisch bei der Entstehung der Tubargravidität eine Rolle zu spielen. Wir sind - auf Einzelheiten an dieser Stelle einzugèhen, würde zı weit führen - von seinen Ausführungen nicht so ganz überzeugt, müssen vielmehr auch für unseren Fall vom rein theoretischen Standpunkte aus die Möglichkeit zugeben, dass das Ei sich in einem Nebentubengang gefangen haben kann. So kann man sich den tiefen Sitz des jungen Eies innerhalb der Ringmusculatur und den Schwund bezüglich die Beiseitedrängung derselben wobl erklären. Allerdings sehr wahrscheinlich ist eine solche Annahme nicht. Denn es hätte sich wohl auf der Längsschnittserie in der nächsten Umgebung des Eies und in der Wand des Eibodens eine Andeutung von einem solchen Gange müssen finden lassen, besonders nach der uterinen Seite zu". Dementsprechend habe ich mich in allen meinen bisherigen Präparaten stets nach Nebentubenlumina umgesehen und namentlich in dem für die allgemeine Auffassung der tubaren Eieinbettung so wichtigen Präparate $\mathrm{II}^{2}$ ) meiner trüheren $A$ bhandlung nichts von solchen gefunden. Der vorliegende Fall ist unter zahlreichen, die ich untersucht habe, der erste, in dem ich ein Nebentubenlumen und Eielemente darin habe nachweisen können. Es erscheint mir dabei von Bedeutung, dass die Zottenbildung erst in einem Bereiche auftritt, das vom Uterus abdominalwärts gerechnet, jenseits der Vereinigungsstelle des Nebentubenganges mit dem Hauptlumen beginnt, so dass in ersterem nur Chorion laeve sich ausbreitet. Inwieweit thatsächlich das $\mathrm{Ei}$ in dem Nebentubengang sich eingebettet hat, ist nicht mehr zu erweisen, da die Schwangerschaft schon zu weit vorgeschritten ist. Es kann jedenfalls auch so sein, dass der Nebentubengang nur in den Bereich des Eies bei seiner weiteren Entwicklung hineinbezogen ist, so dass der vorliegende F'all keinerlei Beweismittel nach der einen oder anderen Richtung bietet. Wohl aber zeigt er an Stellen, die von jeglicher Zerstörung frei sind, wie der epithe-

1) Monatsschr. f. Geb. u. Gyn. Bd. VIII. S. 608.

2) Dieses Archiv. Bd. 63. 1. c. 
liale Ueberzug des Chorion laeve in das subepitheliale Bindegewebe von gegenüberliegenden Tubenfalten eindringt, nachdem er die Cylinderzellen der Tubenschleimhaut auseinandergedrängt hat. In ähnlicher Weise muss sich auch das primäre Eindringen des Eies bis unter das Tubenepithel vollziehen, und da ein derartiger Vorgang beim Menschen in der Tube wohl so leicht nicht der mikroskopischen Untersuchung zugängig werden wird, so giebt Fig. 4, Tafel $\mathrm{V}$ immerhin eine gewisse Vorstellung von einem solchen Lindringen. Letzteres erfolgt in vorliegendem Präparate seitens des Chorion laeve zu einer Zeit, wo die Placenta schon vollständig angelegt ist. Angesichts dieser Thatsachen kann man wohl nicht bestreiten, dass man von einem aggressiven Wachsthum sprechen darf, und wenn dies schon dem Chorion laeve zukommt, um wie viel mehr dann dem Chorion frondosum, d. h. mit anderen Worten: Das Ei gelangt in der Tube nicht nur selbstständig unter das Epithel wie im Uterus, sondern es ist auch weiterhin bei seiner Einriehtung in dem tubaren Fibette activ thätig. Hierbei hat die Finbettung in einem Nebentubengang je nach dessen Lage und je nach dem Orte der Ansiedelung in demselben die Bedeutung, dass das Ei die Grenzen der tubaren Gewebe schneller erreicht and damit die Aussichten für seine weitere Entwicklung von vornherein noch viel ungünstiger gestaltet.

\section{Erklärung der Abbildungen auf Tafel V.}

Figur 1. Rechtsseitiger tubarer Fruchtsack in der Ansicht von vorne. $R$ rom Zuschaver aus das uterine Ende der Tube; auf der Mitte der vorderen Wand ragt Placentargewebe heraus, ebenso aus dem Ostium abdominale; nach unten zu liegt die Abtrennungsstelle vom Lig. latum.

Figur 2-4. Ch E Chorionepithel, T. E Tubenepithel, A E Amnionepitlsel, A $\mathrm{H}$ Amnionhöhle.

Figur 3. a und b Stelien, an denen das Chorionepithel sich zwischen das Tubenepithel einsenkt, c Fältelung des Chorion.

ligur 4. Giebt die Stelle b in Fig. 3 bei starker Vergrösserung wieder. 